\title{
Effects of a sensory-motor orthotic on postural instability rehabilitation in Parkinson's disease: a pilot study
}

\author{
Daniele Volpe ${ }^{1 *}$, Elisa Pelosinn ${ }^{2}$, Leila Bakdounes ${ }^{1}$, Stefano Masiero ${ }^{3}$, Giannettore Bertagnoni ${ }^{4}$, Chiara Sorbera ${ }^{1}$ \\ and Maria Giulia Giantin ${ }^{1}$
}

\begin{abstract}
Background: Proprioceptive deficits have been largely documented in PD patients, thus external sensory signals (peripheral sensory feedback) are often used to compensate the abnormalities of proprioceptive integration. This pilot study aims to evaluate the feasibility and the effectiveness of a rehabilitation-training program, combined with the use of a sensory-motor orthotic in improving balance in a small sample of PD patients.

Methods: Twenty PD patients were randomly allocated into two groups: (i) the Experimental group, where participants were asked to wear a sensory-motor orthotic during the balance training program and (ii) the Control group, where subjects performed an identical training program without wearing any kind of orthotics. In all, the training program lasted 10 sessions (5 days a week for 2 weeks) and the clinical and instrumental assessments were performed at baseline, immediately after the end of the training and 4 weeks after the rehabilitative program was stopped.

Results: All clinical outcome measures tested improved significantly at post and follow-up evaluations in both groups. Interestingly, at the end of the training, only the experimental group obtained a significant improvement in the functional reaching test (sway area - eyes closed) measured by means of stabilometric platform and this result was maintained in the follow-up evaluation.
\end{abstract}

Conclusions: Our preliminary results suggested that the use of a sensory-motor orthotic, in combination with a tailored balance training, is feasible and it seems to positively impact on balance performance in Parkinson's disease.

Trial registration: EudraCT N. 003020-36 - 2013.

Keywords: Parkinson's disease, Sensory-motor orthotic, Postural instability, Rehabilitation

\section{Background}

Parkinson's disease (PD) is a neurological progressive disorder characterized by balance dysfunctions, often associated with the high risk of falling [1] that negatively impacts on the quality of life [2]. In PD, most of the falls occur during a sudden change of posture or during walking [3] in various circumstances (i.e., gait initiation, dual task conditions). Balance problems, in PD patients, are probably due to the overlapping of different factors, such as stopped posture, deficits in postural responses [4], reduced limit of stability [5] and impaired executive

\footnotetext{
* Correspondence: dott.dvolpe@libero.it

'Department of Physical Medicine and Rehabilitation, Neurorehabilitation Unit "Villa Margherita,", Via Costacolonna n.6 Arcugnano, Vicenza, Italy Full list of author information is available at the end of the article
}

function (i.e., deficit in selective attention) [6]. Although much is known about the multifactorial nature of gait disturbances and falls in PD, the pathophysiology of postural instability is still unclear. It seems to depend on a complex interactions between the impairment caused by the disease at different levels of the nervous system and compensatory strategies $[7,8]$. It is well- known that postural control in PD patients mainly relies on visual information, which is possibly used for compensating proprioceptive impairments $[9,10]$. Indeed, PD patients seem to have somatosensory abnormalities with abnormal proprioceptive (kinesthetic) processing that produces a reduced perception of passive motion limb position [11, 12] and space orientation [13]. Therefore, abnormalities in sensory processing have been suggested 
to play a major role in the pathogenesis of sensory dysfunctions in PD [14]. Some authors demonstrated that in a gravity environment, healthy subjects mainly rely on somatosensory information in order to maintain an upright posture [15] and that artificially impairing proprioception worsens postural stability, particularly reducing the COP displacements in response to external perturbations during visual deprivation [16]. In fact, in PD, a defective scaling and habituation of postural reactions during either neck or leg vibration has been revealed $[17,18]$.

Beside the poor effect of dopaminergic treatment in improving balance problems, the effects of physical activity and exercise programs on improving balance [19-21] and quality of life [21] have been extensively proven in patients with PD. However, the possibility of enhancing training effects, by combining intervention with proprioceptive orthotic, has never been tested.

Proprioceptive rehabilitation aims to improve or enhance the perception of proprioceptive signals and their central integration, thus possibly compensating the impaired "gating" function of the basal ganglia [22]. Furthermore, external sensory signals (peripheral sensory feedback) can be used to compensate the abnormal sensorimotor integration in PD patients [23]. Moreover, muscle spindle endings respond to proprioceptive stimulations with an increased muscular activation, thus producing a tonic contraction on the stimulated muscle [24, 25].

In detail, the sensory-motor (SM) orthotic [Fig. 1] used in this study, combines biomechanical and sensorymotor input on the plantar surface of the feet by modulating through function activation of specific muscle groups. In fact, it has been demonstrated that tendon stimulation has an influence on muscular tone with increased voluntary activation and improved muscle velocity and strength $[26,27]$. The proposed novel orthotic is composed of four spots, which through muscle tendon stimulation exerts a compression which activates anticipated muscle contractions: a) the medial spot which activates the medial muscular kinetic chain (tibia, adductor, paraspinal muscles); b) the lateral spot which activates the lateral muscular kinetic chain (peroneal, abductor, iliotibial, paraspinal muscles muscles); c) the metatarsal and under digital spots which stimulate the extensor muscular kinetic chain (fingers flexors, triceps, femoris biceps s, gluteus and paraspinal muscles). No prior study of SM orthosis on balance dysfunctions in PD has been published before. We have no evidence to support this hypothetical mechanism of function.

The present study aims (i) to explore the feasibility and the safety of using a Sensory-Motor orthotic as a tool of increasing plantar proprioceptive information and (ii) investigating if the combination of the SM orthotic, with a balance training, might enhance postural control, balance and gait in a small group of PD patients.

\section{Methods \\ Participants}

A total of 30 patients with idiopathic PD, according to the United Kingdom Parkinson's Disease Society Brain Bank criteria [28], were recruited from the Department of Neurorehabilitation in Villa Margherita, Arcugnano (Vicenza), Italy.

Participants were enrolled in the study if they met the following inclusion criteria: stage 3 of the Hoehn and Yahr (H\&Y) scale, Mini Mental State Examination (MMSE) [29] with score $>24$, ability to walk independently without a walking aid and to attend a physiotherapy venue, the absence of serious co-morbidities (cardiac, pulmonary or orthopaedic diseases) that could impact gait or balance. Patients were excluded if they suffered from major depression (diagnosed by means of a Diagnostic and Statistical Manual of Mental Disorders - DSM V criteria), had Deep

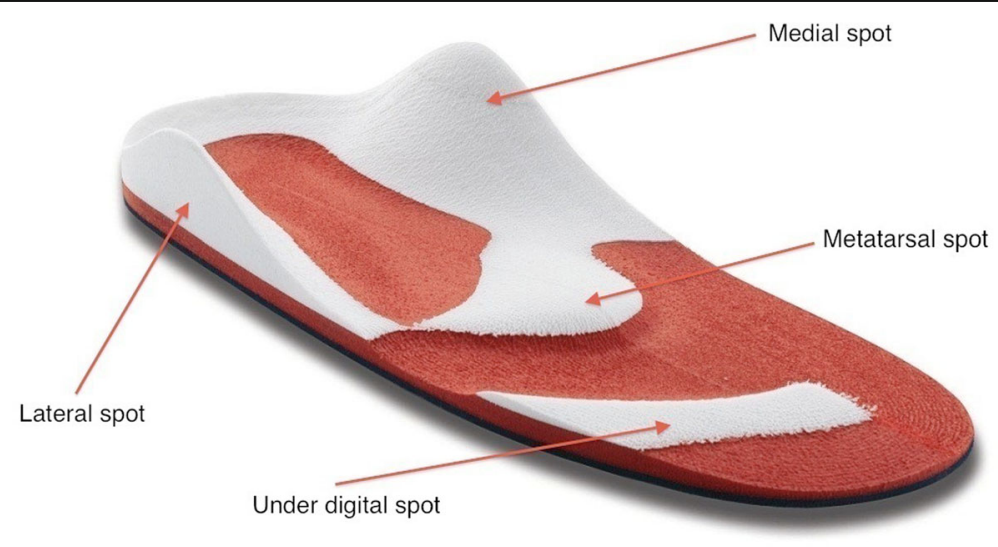

NOVEL ORTHOTIC USED IN THE STUDY

Fig. 1 Example of the sensory-motor orthotic 
Brain Stimulation implants, were medically unstable or had medication induced (dyskinesias), had an history of other conditions affecting stability (e.g., poor visual acuity or vestibular dysfunction, neuropathy or sensory ataxia). In this pilot study, we recruited patients in stage 3 of H\&Y scale exclusively. Thus, all patients were in a moderate stage of PD and had balance problems probably due to abnormal sensory motor integration. In addition, as this was a pilot study, we selected only PD in $H \& Y=3$ because we wanted to limit, as much as possible, the heterogeneity amongst the patients recruited. At the end of the screening phase, twenty patients with PD were enrolled in the study and ten patients were excluded because six participants did not meet the inclusion criteria $(n=1$ had MMSE > 24; $n=2$ needed assistance during walking; $n=2$ had DBS and $n=1$ had severe dyskinesia) and four patients were unable to attend the physiotherapy program due to personal reasons.

\section{Study design (Fig. 2)}

In this pilot study, after the initial screening procedures, participants were randomly allocated into two groups: (i) The Experimental group, in which participants were asked to wear a SM orthotic before and after the training program or (ii) The Control group, where subjects performed an identical training program without wearing any kind of orthotics.
All the clinical and instrumental assessments were performed at baseline (PRE - within 1 week before the beginning of the intervention), after the end of the training (POST - within two days after the last training session) and 4 weeks after the completion of the rehabilitative program (FU - follow-up assessment). Randomization procedure, conducted by a third party, was used to allocate participants to one of the two treatment groups (i.e., experimental or control groups). The assessors were blinded to the group allocation during the whole duration of the study. The study coordinator responsible for the SM orthotics supervision was not blinded to the group allocation, but he was not involved in rehabilitation procedures or outcome assessments. The physiotherapists providing the training program were blinded and not involved in other aspects of the trial (i.e., aims, hypothesis or predictions of the study were not disclosed).

\section{Interventions}

All PD subjects underwent a training balance program composed by 10 sessions ( 5 days a week for 2 weeks). Each session lasted $50 \mathrm{~min}$ and the exercises were identical for both groups. Table 1 details the type of daily balance training program provided by the hospital physiotherapists in accordance to the Koninklijk Nederlands Genootschap voor Fysiotherapie - KNGF Guidelines for Physiotherapy. At the beginning of each session, participants were

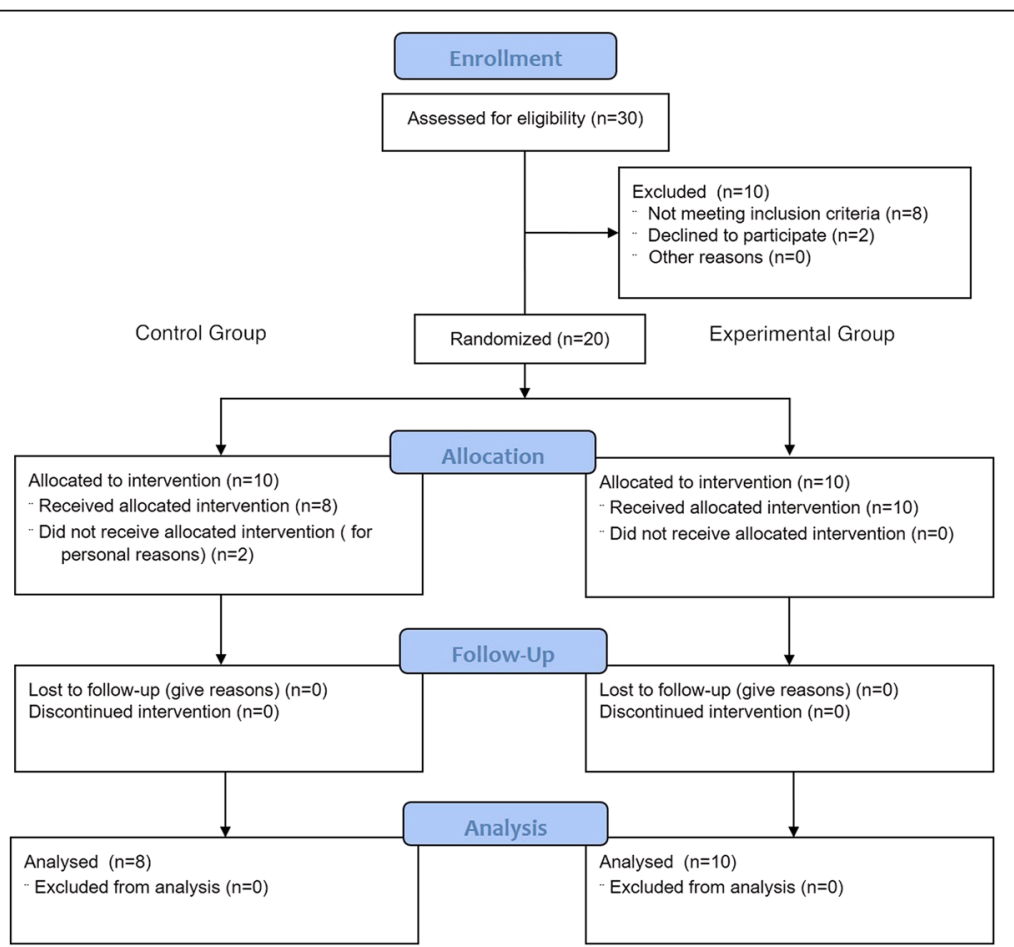

Fig. 2 CONSORT 2010 Flow Diagram. Effects of a Sensori-Motor Orthotic on Rehabilitation of Postural Instability in Parkinson's disease: a Phase II Randomized Pilot Study 
Table 1 KNGF Guidelines: physiotherapy program for balance training

$\begin{array}{ll}\begin{array}{l}\text { Improvement of physical } \\ \text { capacity }\end{array} & \begin{array}{l}\text { To maintain or to improve physical capacity with training of aerobic muscle strength (with the emphasis on } \\ \text { the muscles of the trunk and legs), joint mobility (among others, axial) and muscle length (among others, } \\ \text { muscles of the calf and the hamstrings, flexor and extensor of the knee) }\end{array} \\ \begin{array}{l}\text { Improvement of the transfers } \\ \text { Normalizing body posture }\end{array} & \begin{array}{l}\text { To train transfers by applying cognitive improvement strategies and cues to initiate and continue movements } \\ \text { To prevent or treat postural deformities with exercises for postural alignment and coordinated movements }\end{array} \\ \begin{array}{l}\text { To optimize balance during the performance of activities in static and dynamic conditions with exercises for } \\ \text { training strength and perturbation-based balance training with emphasis of functional reaching test in protected } \\ \text { condition and how to activate postural responses to perturbation. Falls prevention strategies. }\end{array} \\ \begin{array}{l}\text { To walk safely and to increase (comfortable) walking speed with exercise walking with the use of cues and } \\ \text { cognitive movement strategies and to train muscle strength and mobility of the trunk and upper and lower limbs. }\end{array}\end{array}$

required to sign a form in order to attest their attendance. The physiotherapy protocol included $30 \mathrm{~min}$ of exercises designed to improve balance. Precisely, intervention included a perturbation-based balance-training program, where patients were asked to voluntarily reach their limit of stability. During these exercises, participants were required to concentrate and activate the appropriate postural responses in order to react to the external perturbations. Balance training was preceded by warming up and stretching exercises and followed by a cooling down period. Each phase lasted approximately $10 \mathrm{~min}$. Subjects who enrolled in the Experimental group were required for the entire duration of the study (2 weeks) to wear the SM orthotics all day long except during the training balance sessions.

\section{Clinical and instrumental tests Clinical assessments}

Motor impairment was assessed during the III section (motor examination) of the Unified Parkinson's disease Rating Scale (UPDRS) [30], the Berg Balance Scale (BBS) [31], the Timed Up and Go (TUG) [32], and the Sixminute Walking Test (6mWT) [33]. We also quantified the health-related quality of life in all participants using the Parkinson's Disease Questionnaire (PDQ-39) [34]. All adverse events such as injuries, distress and hospital admissions were verified by phone interviews and recordings taken during the pilot study period.

\section{Posturography assessments}

Static posturography was assessed in keeping with current guidelines [35]. The Center of Pressure (CoP) excursion was recorded by means of a force platform (Milletrix model 2.0-Rome, Italy). All data were collected with a $50 \mathrm{~Hz}$ sampling frequency. The CoP was recorded during an upright stance in a quiet room. Participants were instructed to stand erect, with their arms alongside their body. Their feet were kept at an angle of about $30^{\circ}$ opened to the front and with the heels approximately $3 \mathrm{~cm}$ apart. Furthermore, an instrumental version of the functional reaching test (FRT) [36] was executed by asking the subject to elevate their arm to shoulder's height and then to perform a maximum forward reach, while maintaining the heel on the platform with their feet planted in a standing position.

In all the tasks, data was collected for $51.2 \mathrm{~s}$, in both eyes opened (EO) and eyes closed (EC) conditions. The following parameters were taken into account: the sway area $\left(\mathrm{mm}^{2}\right)$, measured as the 95th percentile of an ellipse fitted to the overall CoP trace; COP velocity $(\mathrm{mm} / \mathrm{s})$ and the Romberg index. These parameters were chosen as a tool to evaluate CoM displacement during sway as a response to perturbation.

\section{Statistical analysis}

Demographic and clinical characteristics between the two intervention groups of PD (Experimental and Control) were tested by means of Chi-square $\left(\chi^{2}\right)$ test (gender) and $t$-test (age, UPDRS - Part III Motor, and disease duration) statistics. All clinical and instrumental variables were examined for normality (Shapiro-Wilk W test), and mean and standard deviation (SD) were calculated. For the analysis a Repeated Measures (RM) Analysis of Variance (ANOVA) was used with Group (Experimental, Control) as between-subjects factor and Time (Baseline, Post and Follow-up) as within-subjects factor. The pre-defined level of significance was set at $p<0.05$. Post hoc analysis of significant interactions was performed by means of -tests applying the Bonferroni correction for multiple comparisons when necessary. All statistical analyses were performed with SPSS 22.0.

\section{Results}

At the end of the study, two patients were excluded from the analysis because they dropped out from the training protocol due to personal reasons. Patients with PD enrolled into two groups, did not differ for demographic, clinical characteristics (Table 2) and clinical assessment ( $p$ always $>0.05$ ) recorded at the baseline. For the sample as a whole, $100 \%$ of intervention sessions were delivered across study arms. No major adverse event or death was recorded during the study period. 
Table 2 Baseline demographic and clinical variables of the two groups enrolled in the study

\begin{tabular}{llll}
\hline & $\begin{array}{l}\text { EXP Group } \\
\text { mean } \pm \text { SD }\end{array}$ & $\begin{array}{l}\text { CTRL Group } \\
\text { mean } \pm \text { SD }\end{array}$ & $\begin{array}{l}\text { Statistics } \\
\text { Baseline }\end{array}$ \\
\hline Gender (M/F) & $7 / 3$ & $5 / 3$ & \\
Age (yr) & $69.18 \pm 7.61$ & $63.37 \pm 6.89$ & $p=0.24$ \\
Height (cm) & $160.91 \pm 9.58$ & $160.62 \pm 14.74$ & $p=0.96$ \\
Weight (kg) & $69.54 \pm 13.33$ & $67.62 \pm 8.31$ & $p=0.72$ \\
Disease duration (yr) & $7.82 \pm 4.00$ & $8.12 \pm 2.90$ & $p=0.86$ \\
Falls (n) & $1.45 \pm 2.16$ & $0.87 \pm 0.99$ & $p=0.07$ \\
Levodopa (mg/day) & $455.32 \pm 355.49$ & $409.19 \pm 340.68$ & $p=0.74$ \\
- Dopamine agonist (LEDD mg) & & \\
Pramipexole E.R. & $n=2$ & $n=3$ & N.A. \\
Ropirinole E.R. & $n=3$ & $n=3$ & N.A. \\
Rotigotine ( $n=1)$ & $n=1$ & $n=1$ & N.A. \\
Rasagiline ( $n=1)$ & $n=2$ & $n=1$ & N.A. \\
- Other drugs (LEDD mg) & & & \\
Entacapone & $n=1$ & $n=2$ & N.A. \\
Selegiline & $n=1$ & $n=2$ & N.A. \\
Amantadine & $n=2$ & $n=2$ & N.A. \\
\hline
\end{tabular}

Exp, Experimental; CTRL, Control; M, Male; F, Female; Yr, Years; Cm, centimeters; Kg, Kilograms; $\mathrm{Mg}=$ Milligrams; $\mathrm{N}$, number; $\mathrm{ER}=$ Extended Released; N.A., Not Applicable

\section{Clinical assessments}

All data [mean \pm standard deviation (SD)] collected at baseline, post and follow-up examinations are reported in Table 3. Statistical analysis showed a positive effect of the balance-training program with no differences between groups in all the variables considered. Precisely, the mean score of UPDRS-III was significantly reduced in the Experimental as well as in the Control groups. RM-ANOVA revealed a significant effect of TIME $(p<0.01)$, without any significant Time $\mathrm{X}$ Group interaction $(p=0.41)$. Interestingly, improvements were seen both immediately after the training and at the FU examination ( $\mathrm{p}$ always $<0.01$ ).

For the tests assessing dynamic balance performance (BBS and TUG), RM-ANOVA showed a main effect of TIME (BBS: $p<0.01$ and TUG: $p<0.01$ ) with no Time $\mathrm{X}$ Group interaction. In details, for BBS a significant increase of the total score was seen at Post $(p<0.01)$ and at the FU $(p<0.01)$ evaluations as well as for TUG, where a significant decrease of time in performing the test was seen immediately after the training $(p=0.01)$ and 1 month later (FU: $p<0.01$ ). No Time X Group interaction was revealed by the statistical analysis. Similar results were also found in gait resistance performance. Indeed, the analysis of 6MWT data showed a significant effect of Time $(p=0.02)$ with no differences between the two groups. Thus, an overall improvement was seen immediately after the training (Post: $p=0.03$ ) and it was maintained at the FU examination $(p=0.01)$. Balance and gait improvements were also confirmed by a significant decrease of fall rate. Indeed, RM-ANOVA showed a main effect of Time $(p<0.01)$ with an improvement at post $(p=0.01)$. However, no significant Time $\mathrm{X}$ Interaction was recorded by the statistical analysis $(p=0.55)$. Finally, positive changes on participants' QoL recorded by means of PDQ-39 questionnaire were seen at the end of the training (Post: $p=0.03$ ) as well as the following testing time (FU: $p=0.02$ ). Indeed, RMANOVA revealed a significant effect of TIME $(p=0.02)$ with no significant Time X Group interaction.

\section{Posturography}

Statistical analysis did not reveal significant changes for sway area recorded in the quiet stance test ( $\mathrm{p}$ always $>0.05$ ) in both conditions (EC and EO). However, RM ANOVA showed a significant main effect of Group $(p=0.04)$ and a significant Group x Time interaction $(p=0.03)$ for $95 \%$ confidence ellipse area data obtained during the FRT test in the EC condition. Furthermore, post-hoc analysis revealed that only the experimental group obtained a significant improvement at the end of the training period $(p=0.02)$ and this result was maintained at the follow-up examination (Fig. 3). Similar results were also found for the values obtained for the Romberg index. Indeed, statistical analysis (RM-ANOVA) revealed a significance of the factor Group $(p=0.04)$ as well as a significant Group x Time interaction. Post-hoc analysis showed that only in the experimental group, velocity increased at the end of the training $(p=0.03)$ and at the follow-up evaluation $(p=0.04)$ (Fig. 4). No significant changes were detected during static and dynamic (FRT) evaluation under EO condition. Finally, no significant changes were found for $\mathrm{CoP}$ velocity in any experimental condition (EC and $\mathrm{EO}$ ).

\section{Discussion}

The aim of the present study was to explore the feasibility and the safety of using a Sensory-Motor orthotic as a tool for increasing plantar proprioceptive information. Furthermore, it was carried out to verify if the combination of the SM orthotic, with a rehabilitative intervention, could enhance postural control, balance and gait in a group of subjects with PD.

The rehabilitative program was delivered successfully, with a good level of adherence rate confirmed by the patient's participation and involvement. On the whole, our results demonstrated that combining balance training with a sensory-motor orthotics in a rehabilitation setting is feasible and might lead to some clinically meaningful effect in PD patients with postural instability. However, only subjects enrolled in the experimental protocol 
Table 3 Clinical variables of the two groups enrolled in the study and their comparisons at each time point

\begin{tabular}{|c|c|c|c|}
\hline & PSM Group & CTRL Group & $\begin{array}{l}\text { Statistic } \\
\text { post-hoc TIME }\end{array}$ \\
\hline Motor UPDRS section III at T0-Baseline & $40.87 \pm 6.01$ & $39.00 \pm 11.89$ & \\
\hline Motor UPDRS section III at T1-Discharge & $37.12 \pm 6.66$ & $36.90 \pm 12.02$ & $p<0.01$ \\
\hline Motor UPDRS section III at T2-Follow up & $35.55 \pm 6.57$ & $36.80 \pm 11.80$ & $p<0.01$ \\
\hline Berg Balance Scale T0-Baseline & $45.63 \pm 5.92$ & $45.12 \pm 4.58$ & \\
\hline Berg Balance Scale T1-Discharge & $49.3 \pm 3.15$ & $47.12 \pm 5.05$ & $p<0.01$ \\
\hline Berg Balance Scale T2-Follow up & $50.1 \pm 2.72$ & $49.37 \pm 5.35$ & $p<0.01$ \\
\hline Falls T0-Baseline & $1.45 \pm 2.16$ & $0.87 \pm 0.99$ & \\
\hline Falls T1-Discharge & $0.45 \pm 1.03$ & $0.12 \pm 0.31$ & $p<0.01$ \\
\hline Falls T2-Follow up & $0.00 \pm 0.00$ & $0.00 \pm 0.00$ & N.A. \\
\hline Timed Up and Go T0-Baseline & $13.08 \pm 2.17$ & $13.8 \pm 3.43$ & \\
\hline Timed Up and Go T1-Discharge & $12.13 \pm 1.35$ & $12.8 \pm 2.81$ & $p=0.01$ \\
\hline Timed Up and Go T2-Follow up & $10.81 \pm 1.07$ & $13.2 \pm 2.75$ & $p<0.01$ \\
\hline 6MWT T0-Baseline & $305.64 \pm 48.89$ & $319.8 \pm 48.59$ & \\
\hline 6MWT T1-Discharge & $335.64 \pm 44.09$ & $332.5 \pm 66.00$ & $p=0.03$ \\
\hline 6MWT T2-Follow up & $342.2 \pm 59.99$ & $328.38 \pm 70.18$ & $p=0.01$ \\
\hline PDQ-39 T0-Baseline & $57.7 \pm 22.93$ & $59 \pm 14.38$ & \\
\hline PDQ-39 T1-Discharge & $54.36 \pm 24.47$ & $49.5 \pm 20.52$ & $p=0.03$ \\
\hline PDQ-39 T2-Follow up & $52.1 \pm 27.44$ & $51.25 \pm 19.46$ & $p=0.02$ \\
\hline
\end{tabular}

Exp, Experimental, CTRL Control, UPDRS Unified Parkinson Disease Rating Scale, 6MWT Six Meters Walking Test, PDQ-39 Parkinson's Disease Questionnaire-39 items. N.A., not applicable

$P$ values represent the post hoc analysis (T0 vs T1 and T0 vs T2) when a main effect of TIME was detected with Repeated Measures ANOVA

significantly improved their limit of stability measured by a stabilometric platform. Precisely, an increase of sway area values, obtained during the instrumental functional reaching test, and an improvement of the Romberg index were seen only in the experimental group immediately after the training and follow-up evaluation. As stated in the introduction, PD-related abnormality in proprioception might manifest itself as alteration of kinesthesia (for a review see [13]. Indeed, PD patients have an impaired sense of the timing [37] and discrimination [38] of proprioceptive inputs, which can also lead to deficient compensation of mechanical perturbations, especially during the activation of anticipatory postural adjustments [39]. The enhancement of the proprioceptive inflow, as that induced by the sensory-motor orthotic used in this study, might overcome the subtle impairment in kinesthesia, as previously argued [37]. PD patients used to have a reduced limit of stability particularly during dynamic conditions, thus pointing to dynamic posturography as a better instrument of capturing improvements in

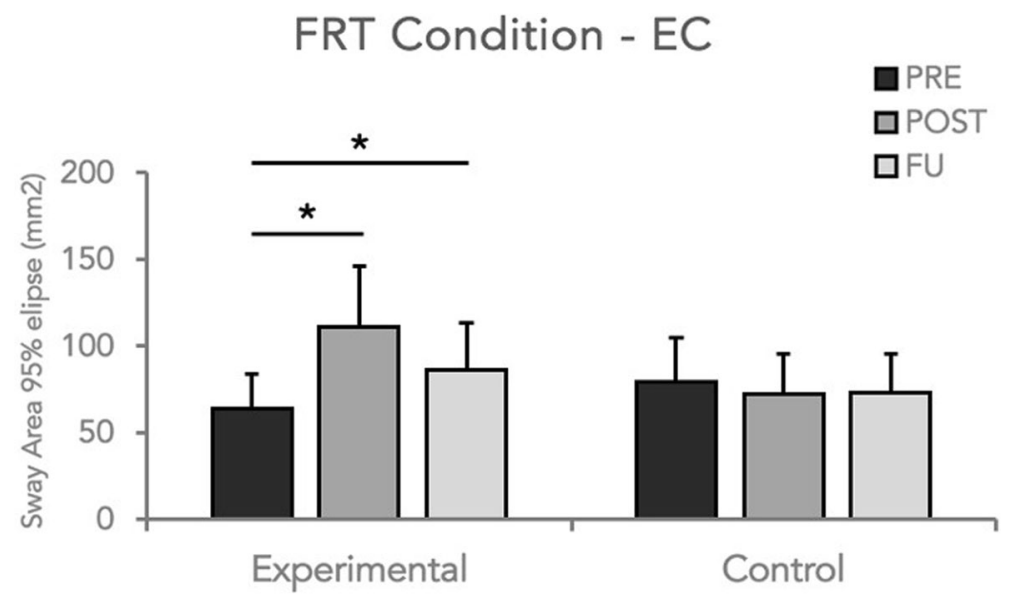

Fig. 3 Sway area values during instrumental FRT-EC condition of the two groups enrolled in the study at each time point 


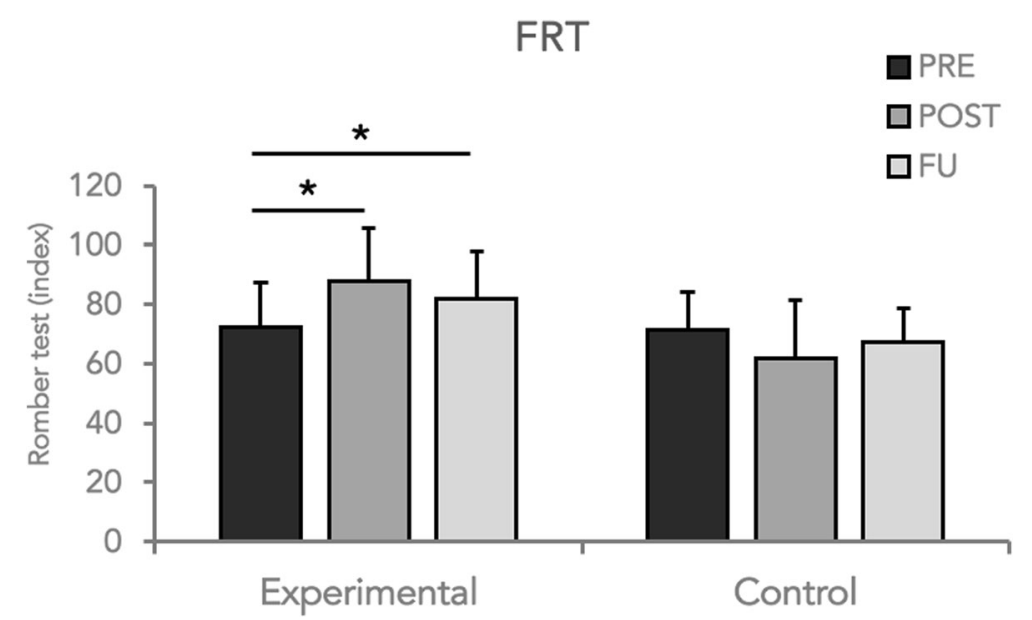

Fig. 4 Romberg index values during FRT condition of the two groups enrolled in the study at each time point

balance [5, 35]. It is well-known that anticipatory postural adjustments and reactive postural reactions in PD are compromised, in the sense that they are reduced in amplitude and velocity [39]. So another possible mechanism of action could be related to the influence on muscles of proprioceptive stimulation exerted by the SM orthotic, since tendon stimulation $[40,41]$ seems to increase muscular tone and velocity promoting the activation of anticipatory postural adjustments and reactive postural reactions. Finally, it is important to notice that significant changes in the posturographic data during the FRT in the experimental group were seen only when patients were required to execute the test with their eyes closed, a set-up relying on proprioceptive information. This fact might suggest an improvement of proprioceptive signals derived from the effect of the SM orthotic.

This pilot study has a number of limitations. Firstly, even if testing occurred at the peak dose of the morning medications, we cannot rule out the bias introduced by fluctuations in levodopa plasmatic concentration. Secondly, even though the sample size allowed the detection of significant changes, here we reported results obtained in a small group of patients, thus our results have to be replicated by larger trials. Thirdly, due to the shortness of training and the follow-up examination, we did not evaluate changes in fall rates. Further study should have to include episode supervision of falls. Fourthly, even if the physiotherapy program for balance training was conducted in accordance with published guidelines, the execution of exercises were influenced by therapists expertise and patients' motivation, meaning that our protocol does not necessarily reflect the clinical practice in other parts of the world. Fifthly, we did not include in this pilot study, an aged matched control group for evaluating changes in balance related to basal ganglia dysfunction, so we cannot conclusively ascribe our findings to basal ganglia malfunction in PD.
Finally, we want to underline that postural control measured by dynamic posturography might give more information about mechanisms of postural instability in PD than static posturography. Performing the FRT might not be as good as a test measured by dynamic posturography.

\section{Conclusions}

This pilot study shows that a tailored balance training, in association with the sensory-motor orthotic, appears to be safe and feasible and is able to positively impact on mobility, balance, gait and quality of life. This preliminary study provides promising data on the feasibility and safety of our protocol, thus supporting the development of a large scale Randomized Control Trial. Future studies are certainly needed and will expand our knowledge on the mechanisms of action of SM orthotic, on the time needed to achieve a meaningful improvement and its long-term duration.

\section{Abbreviations}

$6 \mathrm{mWT}$ : Six minutes walking test; ANOVA: Analysis of Variance; BBS: Berg Balance Scale; CoP: Center of Pressure; DSM: Diagnostic and Statistical Manual of Mental Disorders; EC: Eyes closed; EO: Eyes open; FRT: Functional Reaching Test; H\&Y: Hoehn and Yahr; KNGF: Koninklijk Nederlands Genootschap voor Fysiotherapie; MMSE: Mini Mental State Examination PD: Parkinson's disease; PDQ-39: Parkinson's Disease Questionnaire; RM: Repeated Measures; SD: Standard Deviation; SM: Sensory motor; TUG: Time Up and Go; UPDRS: Unified Parkinson's Disease Rating Scale

\section{Acknowledgements}

We acknowledge Sanitaria Elena Ortopedia for providing the SM orthotics used in the study and also the PD patients that gently participated in this trial.

\section{Funding}

This pilot study was unfunded. The research was performed in the course of the author's clinical duties at the Department of Neurorehabilitation, Villa Margherita (VI). 


\section{Availability of data and materials}

The dataset supporting the conclusions of this article is available upon request.

\section{Authors' contributions}

DV conceived the study, participated in the design, oversaw data collection, contributed to data interpretation and results analysis, and drafted the manuscript. EP was responsible of statistical analysis, contributed to data interpretation, results analysis and drafted the manuscript. LB participated in data collection, was responsible for assessments, contributed in manuscript preparation. SM oversaw data collection, contributed to data interpretation and results analysis. GB oversaw data collection, contributed to data interpretation and results analysis. CS participated in data collection, was responsible for assessments, contributed to manuscript preparation. MGG participated in data collection, was responsible for assessments, contributed in manuscript preparation. All authors read and approved the final manuscript.

\section{Competing interests}

The authors declare that they have no competing interests.

\section{Consent for publication}

Not applicable.

\section{Ethics approval and consent to participate}

The study was approved by the hospital ethical committee C.E.I.O.C. ref n. 35/ 2013 and written informed consent was obtained from all the participants prior to the screening phase. This pilot study did not receive any sources of funding.

\section{Publisher's Note}

Springer Nature remains neutral with regard to jurisdictional claims in published maps and institutional affiliations.

\section{Author details}

'Department of Physical Medicine and Rehabilitation, Neurorehabilitation Unit "Villa Margherita,", Via Costacolonna n.6 Arcugnano, Vicenza, Italy. ${ }^{2}$ Department of Neuroscience, University of Genoa, Genoa, Italy. ${ }^{3}$ School of Physical Medicine and Rehabilitation, University of Padua, Padua, Italy. ${ }^{4}$ Department of Physical Medicine and Rehabilitation, S. Bortolo Hospital, Vicenza, Italy.

Received: 29 August 2016 Accepted: 11 May 2017

Published online: 06 July 2017

\section{References}

1. Grimbergen YA, Munneke M, Bloem BR. Falls in Parkinson's disease. Curr Opin Neurol. 2004;17(4):405-15.

2. Soh SE, Morris ME, McGinley JL. Determinants of health-related quality of life in Parkinson's disease: a systematic review. Parkinsonism Relat Disord. 2011;17:1-9.

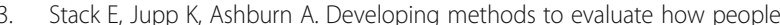
with Parkinson's disease turn 180 degrees: an activity frequently associated with falls. Disabil Rehabil. 2004;26:478-84.

4. Lee RG, Tonolli I, Viallet F, Aurenty R, Massion J. Preparatory postural adjustments in parkinsonian patients with postural instability. Can J Neurol Sci. 1995:22:126-35.

5. Schieppati M, Hugon M, Grasso M, Nardone A, Galante M. The limits of equilibrium in young and elderly normal subjects and in parkinsonians. Electroencephalogr Clin Neurophysiol. 1994;93:286-98.

6. Hausdorff JM, Balash J, Giladi N. Effects of cognitive challenge on gait variability in patients with Parkinson's disease. J Geriatr Psychiatry Neurol. 2003;16:53-8.

7. Fasano A, Plotnik M, Bove F, Berardelli A. The neurobiology of falls. Neurol Sci. 2012;33:1215-23.

8. Benatru I, Vaugoyeau M, Azulay JP. Postural disorders in Parkinson's disease. Neurophysiol Clin. 2008;38:459-65.

9. Demirci M, Grill S, McShane L, Hallett M. A mismatch between kinesthetic and visual perception in Parkinson's disease. Ann Neurol. 1997;41:781-8.

10. Carpenter MG, Bloem BR. Postural control in Parkinson patients: a proprioceptive problem? Exp Neurol. 2011;227(1):26-30.

11. Zia S, Cody F, O'Boyle D. Joint position sense is impaired by Parkinson's disease. Ann Neuro. 2000;47:218-28.
12. Konczak J, Krawczewski K, Tuite P, Maschke M. The perception of passive motion in Parkinson's disease. J Neurol. 2007:254:655-63.

13. Conte A, Khan N, Defazio G, Rothwell JC, Berardelli A. Pathophysiology of somatosensory abnormalities in Parkinson disease. Nat Rev Neurol. 2013;9: 687-97.

14. Nolano M, Provitera V, Estraneo A, Selim MM, Caporaso G, Stancanelli A Saltalamacchia AM, Lanzillo B, Santoro L. Sensory deficit in Parkinson's disease: evidence of a cutaneous denervation. Brain. 2008;131:1903-11.

15. Peterka RJ. Sensorimotor integration in human postural control. J Neurophysiol. 2002;88:1097-118.

16. Mohapatra S, Krishnan V, Aruin AS. Postural control in response to an external perturbation: effect of altered proprioceptive information. Exp Brain Res. 2012:217:197-208.

17. Valkovic P, Krafczyk S, Saling M, Benetin J, Botzel K. Postural reactions to neck vibration in Parkinson's disease. Mov Disord. 2006:21:59-65.

18. Valkovic P, Krafczyk S, Botzel K. Postural reactions to soleus muscle vibration in Parkinson's disease: scaling deteriorates as disease progresses. Neurosci Lett. 2006;401:92-6.

19. Hirsch MA, Toole T, Maitland CG, Rider RA. The effects of balance training and high-intensity resistance training on persons with idiopathic Parkinson's disease. Arch Phys Med Rehabil. 2003;84:1109-17.

20. Allen NE, Sherrington C, Paul SS, Canning CG. Balance and falls in Parkinson's disease: a meta-analysis of the effect of exercise and motor training. Mov Disord. 2011;26:1605-15.

21. Goodwin VA, Richards SH, Henley W, Ewings $\mathrm{P}$, Taylor AH, et al. Anexercise intervention to prevent falls in people with Parkinson's disease: a pragmatic randomised controlled trial. J Neurol Neurosurg Psychiatry. 2011;82:1232-8.

22. Abbruzzese G, Trompetto C, Mori L, Pelosin E. Proprioceptive rehabilitation of upper limb dysfunction in movement disorders: a clinical perspective. Front Hum Neurosci. 2014;8:961.

23. Abbruzzese $G$, Berardelli A. Sensorimotor integration in movement disorders. Mov Disord. 2003;18:231-24060

24. Roll JP, Vedel JP. Kinaesthetic role of muscle afferents in man, studied by tendon vibration and microneurography. Exp Brain Res. 1982;47:177-90.

25. Marsden CD, Meadows JC, Hodgson HJ. Observations on the reflex response to muscle vibration in man and its voluntary control. Brain. 1969;92:829-46.

26. Cordo P, Gurfinkel VS, Bevan L, Kerr GK. Proprioceptive consequences of tendon vibration during movement. J Neurophysiol. 1995;74:1675-88.

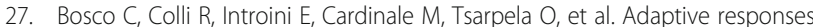
of human skeletal muscle to vibration exposure. Clin Physiol. 1999;19:183-7.

28. Hughes AJ, Daniel SE, Kilford L, Lees AJ. Accuracy of clinical diagnosis of idiopathic Parkinson's disease: a clinico-pathological study of 100 cases. J Neurol Neurosurg Psychiatry. 1992;55:181-4.

29. Folstein MF, Folstein SE, McHugh PR. "Mini-mental state". A practicalmethod for grading the cognitive state of patients for the clinician. J Psychiatr Res. 1975:12:189-98

30. Fahn S, Elton R, et al. Recent developments in Parkinson's disease. Folorham Park: Macmillan Health Care Information; 1987. p. 153-63. 293-304.

31. Berg K, Wood-Dauphinee S, Williams JI. The Balance Scale: reliability assessment with elderly residents and patients with an acute stroke. Scand J Rehabil Med. 1995;27:27-36.

32. Podsiadlo D, Richardson S. The timed "Up \& Go": a test of basic functional mobility for frail elderly persons. J Am Geriatr Soc. 1991;39:142-8.

33. Casanova C, Celli BR, Casas A, Cote C et al. The 6-min walk distance in healthy subjects: reference standards from seven countries. Eur Respir J. 2011;37:137-56.

34. Peto V, Jenkinson C, Fitzpatrick R, Greenhall R. The development and validation of a short measure of functioning and well being for individuals with Parkinson's disease. Qual Life Res. 1995:4:241-8.

35. Scoppa F, Capra R, Gallamini M, Shiffer R. Clinical stabilometry standardization: basic definitions-acquisition interval-sampling frequency. Gait Posture. 2013;37:290-2.

36. Duncan PW, Weiner DK, Chandler J, Studenski S. Functional reach: a new clinical measure of balance. J Gerontol. 1990;45:M192-7.

37. Fiorio M, Stanzani C, Rothwell JC, Bhatia KP, Moretto G, et al. Defective temporal discrimination of passive movements in Parkinson's disease. Neurosci Lett. 2007:417:312-5.

38. Jacobs JV, Horak FB. Abnormal proprioceptive-motor integration contributes to hypometric postural responses of subjects with Parkinson's disease. Neuroscience. 2006;141:999-1009. 
39. Horak FB, Dimitrova D, Nutt JG. Direction-specific postural instability in subjects with Parkinson's disease. Exp Neurol. 2005;193(2):504-21.

40. De Nunzio AM, Nardone A, Picco D, Nilsson J, Schieppati M. Alternate trains of postural muscle vibration promote cyclic body displacement in standing parkinsonian patients. Mov Disord. 2008;23:2186-93.

41. Nonnekes J, de Kam D, Geurts A, Weerdesteyn V, Bloem BR. Unraveling the mechanisms underlying postural instability in Parkinson's disease using dynamic posturography. Expert Rev Neurother. 2013;13:1303-8.

Submit your next manuscript to BioMed Central and we will help you at every step:

- We accept pre-submission inquiries

- Our selector tool helps you to find the most relevant journal

- We provide round the clock customer support

- Convenient online submission

- Thorough peer review

- Inclusion in PubMed and all major indexing services

- Maximum visibility for your research

Submit your manuscript at www.biomedcentral.com/submit 